\title{
\begin{tabular}{ll} 
Research Square & Preprints are preliminary reports that have not undergone peer review. \\
\hline & They should not be considered conclusive, used to inform clinical practice,
\end{tabular} or referenced by the media as validated information.
}

\section{Emodin Protects SH-SY5Y Cells Against Zinc- Induced Synaptic Impairment and Oxidative Stress Through ERK1/2 Pathway}

\section{Qian Chen}

The first affiliated Hospital of Guizhou University of Traditional Chinese Medicine

Chencen Lai

The first affiliated Hospital of Guizhou University of Traditional Chinese Medicine

\section{Fa Chen}

The first affiliated Hospital of Guizhou University of Traditional Chinese Medicine

\section{Yuanting Ding}

The first affiliated Hospital of Guizhou University of Traditional Chinese Medicine

\section{Yiyuan Zhou}

The first affiliated Hospital of Guizhou University of Traditional Chinese Medicine

\section{Songbai Su}

The first affiliated Hospital of Guizhou University of Traditional Chinese Medicine

\section{Ruiqing Ni}

University of Zurich

Zhi Tang ( $\nabla$ tangzhi_2000@hotmail.com )

The first affiliated Hospital of Guizhou University of Traditional Chinese Medicine

\section{Research Article}

Keywords: emodin, ERK1/2 pathway, mitochondrial, oxidative stress, SH-SY5Y cells, synaptic impairment, zinc

Posted Date: August 24th, 2021

DOl: https://doi.org/10.21203/rs.3.rs-829075/v1

License: (c) (1) This work is licensed under a Creative Commons Attribution 4.0 International License. Read Full License

Version of Record: A version of this preprint was published at Frontiers in Pharmacology on February 7th, 2022. See the published version at https://doi.org/10.3389/fphar.2022.821521. 


\section{Abstract}

Background: Zinc is an essential trace element important for the physiological function of the central nervous system. The abnormal accumulation of zinc inside neurons may induce mitochondrial dysfunction and oxidative stress which contributes to many brain diseases. We hypothesized that natural anthraquinone derivative emodin can protect against neurotoxicity induced by pathological concentrations of zinc via Extracellular signal-regulated kinase 1/2 (ERK1/2) signaling pathway, and alleviate the oxidative stress and mitochondria dysfunction.

Results: Human neuroblastoma (SH-SY5Y cells) was treated with zinc sulfate and different concentrations of emodin, and was examined in the changes in the levels of ERK1/2 expressions, oxidative stress (DCFH-DA staining), mitochondrial function (JC-1 staining), and lipid peroxidation (4hydroxynonenal staining), and DNA oxidation (8-Hydroxy-2-deoxyguanosine staining). Emodin ameliorated zinc-induced altered expression level of phosphorylated-ERK1/2 (not total-ERK1/2), and synaptic proteins (presynaptic SNAP25, synaptophysin and postsynaptic PSD95) in SH-SY5Y cells, in a dose-dependent manor. Moreover, emodin inhibited the generation of reactive oxygen substrates, oxidative stress, facilitated the collapse of mitochondrial membrane potential $(\Delta \Psi \mathrm{m})$ in SH-SY5Y cells.

Conclusion: Our results indicated that emodin exerts neuroprotective effects against zinc by normalizing synaptic impairment via decreasing the phosphorylation of ERK $1 / 2$, reducing reactive oxygen substrates and protecting mitochondrial function.

\section{Background}

Zinc is an essential trace element obtained from the diet that regulates the expression of many biological molecules and activation of signalling pathways. Zinc deficiency affects up to 2 billion people worldwide and has profound effects on immune and neurological system functions[1]. In the central nerves system zinc is one of the most abundant oligoelements, involves in the balance of excitatory and inhibitory signals of synapse[2]. During neuronal activity, zinc is released in the form of free ionic $\left(\mathrm{Zn}^{2+}\right)$ from synaptic vesicles. Maintaining the homeostasis of zinc is thus essential for the physiological function of the brain[3]. Excessive zinc in the extracellular fluid has been shown to increase neurotoxicity, induce mitochondrial dysfunction, and oxidative stress. Abnormal increase in the levels of metal ions including $Z^{2+}$, have been found in $A \beta$, form $A \beta-Z n$ complexes[4], which results in a loss of zinc modulatory activity and cognitive deficits in animal models of Alzheimer's disease (AD)[5].

In addition, zinc accumulation has been shown to cause mitochondrial dysfunction and oxidative stress in $\mathrm{AD}[6,7]$ as well as in ischemic stroke models $[8,9]$. Mitochondrial $\mathrm{Zn}^{2+}$ accumulation is a possible trigger of hippocampal ischemic injury[8]. The synergistic interaction between $\mathrm{Zn}^{2+}$ and reactive oxygen species (ROS) has been shown to amplify the ischemic brain injury in rodent model[9] through direct ROS generation or through, mitochondrial $\mathrm{Ca}^{2+}$ uniporter[10]. Recent study indicates that zinc status introduce through Inflammation through NLRP3-mediated pathway[11]. 
Emodin, an anthraquinone derivative, is a major active ingredient of many herbs including Rheum palmatum, Polygonum cuspidatum, Aloe Vera, and Cassia obtusifolia etc[12]. Emodin shows neuroprotective, anti-inflammatory effects in animal models of cerebral ischemia stroke, traumatic brain injury, $A D$, and Parkinson's disease[13-15]. Different signaling pathways have been reported that mediate the effect of emodin such as the Nrf2, Phosphatidylinositol 3-Kinase/Beclin-1/B-Cell Lymphoma 2, and AMP-activated protein kinase signaling pathway[16-18]. Previous studies from our and other groups have reported that emodin demonstrates neuroprotective effect, and can inhibit the neurotoxic effect of $\mathrm{NaF}$ on SH-SY5Y cells via reducing reactive oxygen substrates (ROS) overproduction and oxidative stress [19].the underlying mechanism of emodin remains to be elucidated.

The Extracellular signal-regulated kinase $1 / 2$ (ERK1/2) is activated by neurotrophins and other chemicals an plays an important role in differentiation, survival, structural plasticity, and long-term potentiation of neurons, as well as memory formation in animal models[20]; Emerging evident suggest that ERK1/2 signal pathway is implicated in a number of neurodegenerative diseases with oxidative stress[21]. Aberrant accumulation of activated ERK $1 / 2$ in neurons has been reported in AD brains[22, 23]. Here, we hypothesized that emodin can protect against neurotoxicity induced by pathological concentrations of zinc via ERK1/2 signaling pathway, and alleviate the oxidative stress and mitochondria dysfunction. We exposed human neuroblastoma SH-SY5Y cells to high dose of zinc sulfate, and assessed the effect of emodin on attenuating synaptic impairment, mitochondria function and oxidative stress damage.

\section{Methods}

\section{Antibodies and reagents}

Emodin with purity $>96 \%$ was purchased from the National Institutes for Food and Drug Control (China). Zinc sulfate was purchased from Sigma-Aldrich (USA). The total ERK $(1: 1000)$ and phosphorylated ERK (1:1000) antibodies were purchased from Cell Signaling Technology (Boston, Massachu-setts). The PSD95 (1:1000), SNAP25 (1:1000), synaptophysin (1:1000), 4-Hydroxynonenal (4-HNE, 1:100), and 8hydroxy-2'-deoxyguanosine (8-OHdG, 1:200) antibodies were bought from Abcam (USA). anti-mouse and anti-rabbit secondary antibodies (1:5000) were purchased from Bio-rad (USA). anti-rabbit DyLight-546 and anti-mouse DyLight 488 secondary antibodies were from Invitrogen (California, USA); Reactive oxygen species assay Kit with 2'-7'dichlorofluorescin diacetate (DCFH-DA) for detecting intracellular hydrogen peroxide $\left(\mathrm{H}_{2} \mathrm{O}_{2}\right)$ and oxidative stress and mitochondrial membrane potential assay kit with JC-1 were bought from Beyotime (China).

\section{Cell culture and treatment}

SH-SY5Y cells were cultured in Dulbecco's modified eagle dedium (DMEM)/ nutrient mixture F-12 (F12) supplemented with $10 \%$ Fetal Bovine Serum (FBS) at $37^{\circ} \mathrm{C}$. At $80 \%$ confluence, cells were seeded into 6well culture plates. After serum deprivation overnight, different concentrations of emodin $(10,25$, and $40 \mu \mathrm{M}$ ) were applied to pre-treat the cells for 2 hours in serum-free media. $300 \mu \mathrm{M}$ zinc sulfate was applied for an additional 4hours. 


\section{Cell extraction and western blotting analysis}

After being washed with cold Phosphate-buffered saline (PBS, pH7.4), the cells were lysed with $100 \mu \mathrm{L}$ preheated sodium dodecyl sulfate (SDS) sample buffer and scraped with a rubber policeman. The extract was placed in an Eppendorf tube and boiled for $5 \mathrm{~min}$, cooled on ice immediately. cell lysates $(10 \mu \mathrm{L})$ were run on TGX Stain-Free-FastCast Acrylamide gels (Bio-Rad). The gels were Stain-Free activated for $45 \mathrm{sec}$ and imaged utilizing the ChemiDoc MP imaging system (Bio-Rad, US). The separated proteins were transferred to the polyvinylidene difluoride (PVDF) membranes utilizing the Trans-Blot Turbo Transfer System (Bio-Rad), and the membranes were blocked with $5 \%$ non-fat dried milk in Tris-buffered saline (TBS) for 1 hour. The block solution was then removed from the incubation solution and specific antibody was added for overnight incubation at $4^{\circ} \mathrm{C}$. Immunoblot images were obtained by ChemiDoc MP imaging system. The stain-free signals and immunoblots were evaluated using the ImageLab software (Bio-Rad). Protein expression levels were normalized to the Stain-Free total protein lane density obtained from each gel .

\section{ROS measurements}

For detecting intracellular $\mathrm{H}_{2} \mathrm{O}_{2}$ and oxidative stress, DCFH-DA staining was used. Cells in 24-well plates at a density of $5 \times 104 /$ well were incubated in control media or $300 \mu \mathrm{M}$ zinc sulfate for 4 hours with or without 2 hours pre-treatment of emodin $(10,25$, and $40 \mu \mathrm{M})$. DCFH-DA staining was used to determine changes in intracellular reactive oxygen species (ROS) levels according to the manufacturers' instructions. Briefly, the cells were maintained in DCFH-DA at $37^{\circ} \mathrm{C}$ for 20 minutes, then removed the staining solution and the cells were washed with PBS pH7.4 twice gently. Image analysis was performed using a confocal microscope (Leica SP8, Leica, Germany) and the Image J 1.49V software.

\section{Mitochondrial membrane potential detection}

Mitochondrial membrane potential was measured using a mitochondrial membrane potential probe JC-1 staining dye in SH-SY5Y cells. Briefly, cells in 24-well plates were treated with $300 \mu \mathrm{M}$ zinc sulfate for 4hours with or without pretreatment of emodin $(10,25$, and $40 \mu \mathrm{M})$ for 2 hours. After adding F12 medium/JC-1 working solution (1:1), cells were maintained in a $\mathrm{CO} 2$ incubator for 20 minutes. The staining solution was removed, then the cells were washed twice gently with $\mathrm{JC}-1$ staining buffer. The fluorescence was detected with confocal microscopy (Leica SP8). and the pictures were captured in five fields of each sample in triplicate. The $\Delta \Psi \mathrm{m}$ of SH-SY5Y cells was represented by the ratio of monomeric JC-1 to aggregated JC-1.

\section{Immunofluorescence staining}

SH-SY5Y cells grown on coverslips were treated with $300 \mu \mathrm{M}$ zinc-sulfate for 4 hours with or without pretreatment of emodin $(10,25$, and $40 \mu \mathrm{M})$ for 2 hours. The cells were washed with PBS pH7.4 and fixed with $4 \%$ paraformaldehyde for 20 minutes. After permeated with TBS containing $0.1 \%$ Triton X-100 for 5 minutes, the cells were blocked with $5 \%$ bovine serum albumin (BSA) in TBS for 30 minutes. The cells were then incubated with primary antibodies against 4-HNE (1:100) or 8-OHdG (1:200) overnight at $4^{\circ} \mathrm{C}$, 
anti-rabbit DyLight-546 and anti-mouse DyLight-488 secondary antibodies (1:200) were incubated for 1 hour in the dark. Nuclei were counter-stained with 4',6-diamidino-2-phenylindole (DAPI). Image analysis was performed using a confocal microscope (Leica SP8) and the Image J 1.49V (NIH, US).

\section{Statistical analysis}

One-way ANOVA and Bonferroni post-hoc analysis was used for group comparisons using GraphPad Prism 8 (GraphPad Prism). Data was presented as the mean \pm standard error $(S E M)(n=3)$. Significant was set at $p<0.05$.

\section{Results}

\section{Emodin attenuated the phosphorylation of ERK1/2 in SH- SY5Y cells}

We hypothesized that the emodin effect on the protection of SH-SY5Y cells against zinc via ERK1/2 pathway. We examined the phosphorylated and total expression levels of ERK in SH-SY5Y cell lysate by using western blotting. $300 \mu \mathrm{M}$ zinc sulfate significantly increased the expression levels of phosphorylated ERK1/2 $(p=0.03)$ but not the total ERK1/2 in the treated SH-SY5Y cells compared to control group (Fig. 1a-d). A dose-dependent effect of emodin pre-treatment on phosphorylated ERK1/2 was observed: pre-treatment with emodin at $40 \mu \mathrm{M}$ (but not at 10 or $25 \mu \mathrm{M}$ dose) totally abolished the increase in the levels of phosphorylated ERK1/2 induced by zinc sulfate ( $p=0.02$, vs. Zn-treated group) (Fig. 1a, c). No difference was observed in the level of total ERK1/2 by pre-treatment with emodin at different dose.

\section{Emodin ameliorated synaptic impairment in zinc sulfate- induced SH-SY5Y cells}

To investigate the possible effects of emodin on synaptic function related proteins that affected by the presence of zinc, we examine the changes in the levels of presynaptic terminal proteins (SNAP25 and synaptophysin) and postsynaptic density protein (PSD95). We found that $300 \mu \mathrm{M}$ zinc sulfate treatment significantly reduced the expression levels of presynaptic SNAP25 $(p=0.04)$, synaptophysin $(p=0.01)$ and postsynaptic PSD 95 (approximately $50 \%, p=0.04$ ) in the SH-SY5Y cells compared to control group, respectively (Fig. 2c, d,e). A dose-dependent protection effect of emodin pre-treatment against synaptic damage caused by zinc treatment was observed: Pre-treatment with emodin at $40 \mu \mathrm{M}$ (but not at 10 or 25 $\mu \mathrm{M})$ significantly ameliorated the reduction in the levels of synaptophysin $(p=0.04)$, and SNAP25 $(p=$ 0.03 ) induced by zinc sulfate compared to zinc-treated control group (Fig. 2c, d). Pre-treatment with emodin at $10 \mu \mathrm{M}$ (but not at 25 or $40 \mu \mathrm{M}$ ) significantly ameliorated the zinc-induced reduction in the levels of PSD 95 by approximately $50 \%(p=0.01)$, compared to zinc-treated control group (Fig. 2e). 


\section{Emodin inhibited ROS generation in zinc-induced SH-SY5Y cells}

To detect the zinc-induced oxidative stress and the antioxidant effect of emodin, we assessed ROS production by using DCFH-DA fluorescent staining in SH-SY5Y cells. $300 \mu \mathrm{M}$ zinc sulfate significantly increased the intracellular level of ROS indicated by DCFH-DA fluorescent intensity in the treated SH-SY5Y cells compared to control group $(p=0.009)$. Pre-treatment with emodin showed a dose-dependent effect: at $40 \mu \mathrm{M}$ (but not at 10 or $25 \mu \mathrm{M}$ ) significantly decreased the zinc-induced increase in DCFH-DA fluorescent intensity by approximately $50 \%$ ( $p=0.04$ vs. zinc-treated control group) (Fig. 3a,b).

\section{Emodin reestablished the loss of mitochondrial membrane potential $(\Delta \Psi \mathrm{m})$ in zinc-induced SH-SY5Y cells}

Next we examed whether emodin can ameliorate the mitochondrial dysfunction induced by zinc treatment. We measured the mitochondrial membrane potential $\Delta \Psi \mathrm{m}$ in zinc-treated SH-SY $5 Y$ cells by using JC-1 staining. JC-1 aggregate in the normal mitochondrial matrix emits red fluorescence. Green fluorescence is produced in JC-1 staining of cells following loss of $\Delta \Psi \mathrm{m}$. The ratio of green and red fluorescence was used to indicate the toxicity introduced by inc in mitochondria and the protective effect of emodin. In control groups, JC-1 aggregated in mitochondria and the green/red fluorescence intensity ratio was $0.77 \pm 0.11$. Exposure of SH-SY $5 Y$ cells to $300 \mu \mathrm{M}$ zinc sulfate for 4 hours increase green $/ \mathrm{red}$ fluorescence intensity ratio to $1.85 \pm 0.14$ ( $p=0.0008$, vs. control), implying the collapse of $\Delta \Psi \mathrm{m}$ (Fig. 4).

In the presence of increasing concentrations of emodin, the green fluorescence gradually weakened, while the red fluorescence remained the same, resulting in a reduced green/red fluorescence intensity ratio. The green/red fluorescence intensity ratio from JC- 1 staining was $1.57 \pm 0.03$ at $25 \mu \mathrm{M}$ ( $p=0.04$, vs. zinctreated control group), and $1.44 \pm 0.03$ at $40 \mu \mathrm{M}(p=0.004$, vs. zinc-treated control group), implying the reestablishment of $\Delta \Psi_{\mathrm{m}}$.

\section{Emodin attenuated oxidative stress damage in zinc-induced SH-SY5Y cells}

To measure the potential oxidative damage following zinc-treatment, products of oxidative stress such as 4-hydroxynonenal (4-HNE) from lipid peroxidation and 8-hydroxy-2'-deoxyguanosine (8-OHdG) from DNA oxidation were analyzed via immunostaining (Figs. 5 and 6). The fluorescence intensity of 4-HNE and 8OHdG staining in the SH-SY5Y cells were quantified among the control, zinc-treated group and emodin

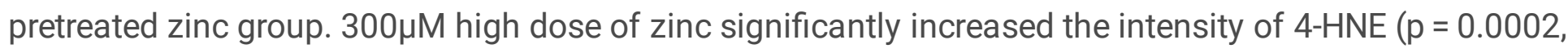
vs. controls). Pre-treatment with emodin at 25 and $40 \mu \mathrm{M}$ (but not $10 \mu \mathrm{M}$ ) significantly reduced the zincinduced increase in the fluorescence intensity of 4-HNE in SH-SY5Y cells by approximately $50 \%(p=$ 0.006 , vs. zinc treated control group) and $70 \%(p=0.0006$, vs. zinc treated control group), respectively, in a dose-dependent manner (Fig. 5a, b). The effect of emodin treatment at $40 \mu \mathrm{M}$ (but not $25 \mu \mathrm{M}$ ) was significantly higher compared to that that $10 \mu \mathrm{M}$ dose $(p=0.005)$ 
High dose of zinc significantly increased the intensity of 8-OHdG $(p=0.002$, compared to the controls. Pre-treatment with emodin at $25,40 \mu \mathrm{M}$ (but not $10 \mu \mathrm{M}$ ) significantly reduced the zinc-induced increase in the fluorescence intensity of 8-OHdG in SH-SY5Y cells by approximately $68 \%(p=0.005$, vs. zinc treated control group) and $74 \%(p=0.002$, vs. zinc treated control group), respectively, with a dose-dependent manner (Fig. 6a, b). The effects of emodin treatment at 25 and $40 \mu \mathrm{M}$ were significantly greater compared to that that $10 \mu \mathrm{M}$ dose $(p=0.03$ and $p=0.01$, respectively $)$.

\section{Discussion}

Our results revealed that treatment with pathological dose $(300 \mu \mathrm{M})$ of zinc cause increase $\mathrm{p}$-ERK $1 / 2$ expression, synaptic impairment, mitochondrial dysfunction and oxidative stress in SH-SY5Y cells. Pretreatment with emodin showed a protective effect against these changes to induced by $300 \mu \mathrm{M}$ zinc in $\mathrm{SH}-\mathrm{SY} 5 \mathrm{Y}$ cells in a dose dependent manner. The concentration of zinc is about $150 \mu \mathrm{M}$ in the brain from healthy controls, whereas the zinc concentrations in $A B$ plaques from brain of $A D$ patients are increased by 3-fold compared with controls (reaching more than $400 \mu \mathrm{M}$ ) [24]. Thus in the present study we used $300 \mu \mathrm{M}$ zinc to mimic the concentration under pathological conditions, which is characterized in detail in previous study[25].

Zinc is also involved in several important signaling pathways, inhibits the proteinphosphatase 2A (PP2A)

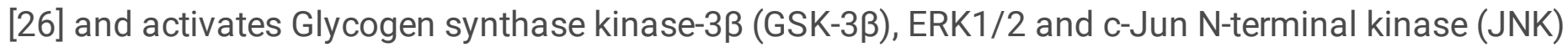
[27-29]. Here we found that zinc-treatment increased the expression of $p$-ERK but not the total ERK, which was entirely abolished by pretreatment with emodin. This implied that emodin may have potential as a therapeutic drug for decreasing high zinc level-induced neurotoxicity via suppressing p-ERK $1 / 2$. In vivo studies in animal models have shown that the chelator-driven perturbation of $\mathrm{Zn}^{2+}$ in brain decreased the levels of brain-derived neurotrophic factor, PSD95, and dendritic spine density[30]. Reduced levels of synaptic-related proteins indicated a declined synaptic function in response to zinc exposure. our results indicated a protective effect exerted by emodin on synaptic impairment, in line with earlier study[19]. However the optimal dosage of emodin against pre- and post- synaptic protein differs in our observation. The highest dose $40 \mu \mathrm{M}$ of emodin significantly alleviated the zinc induced reduction in presynaptic proteins, but did not ameliorated the reduction in post-synaptic PSD 95.

In addition to affecting synaptic function, zinc has been shown linked with oxidative stress as well as neuroinflammation that are important in AD disease development[11]. In the present study, pretreatment of SH-SY5Y cells by emodin suppressed the zinc-induced ROS generation, reduced the formation of lipid peroxidation product (4-HNE), and decreased the level of DNA damage marker (8-OHdG). These results suggest that emodin has an antioxidant effect again zinc-induced ROS generation. The decrease of mitochondrial $\Delta \Psi_{\mathrm{m}}$ is a sign of early apoptosis. In the present study, Zinc-induced ROS generation resulted in a dissipation of $\Delta \Psi \mathrm{m}$, indicating mitochondria dysfunction. The Zinc-induced neurotoxicity has been reported associating with ROS generation[31]. The mitochondrial respiratory chain which regulates apoptosis is susceptible to $\mathrm{Zn}^{2+}$. Accumulation of $\mathrm{Zn}^{2+}$ further induces mitochondrial dysfunction and oxidative stress[32]. Free $\mathrm{Zn}^{2+}$ induces mitochondrial permeability transition and 
generation of ROS[33]. ROS can, in turn, increase the detrimental amount of zinc release from metallothioneins[34], forming a vicious circle. Liu et al have previously reported that emodin inhibited the influx of $\mathrm{Zn}^{2+}(200 \mu \mathrm{M})$ into neuronal cells, thereby preventing the consumption of NAD + and ATP, inhibiting the generation of ROS and ER stress, and inactivating AMPK/ACC signaling pathways to exert neuroprotective effects[35].

\section{Conclusions}

In conclusion, we demonstrated a protective effect of emodin against zinc-induced neurotoxicity in SHSY5Y cell line. Emodin pretreatment normalized the zinc-induced synaptic impairment, reduced the oxidative stress through the inhibition of phosphor-ERK1/2 signal pathway, and inhibited mitochondrial dissipation in a dose-dependent manner. Further studies are needed to investigate neuroprotective effects of emodin and pathways involved in animal models with pathological accumulation of zinc in brain.

\section{Abbreviations}

ERK1/2: Extracellular signal-regulated kinase 1/2; $\Delta \Psi \mathrm{m}$ : Mitochondrial membrane potential; AD: Alzheimer's disease; ROS: Reactive oxygen species; 4-HNE:4-hydroxynonenal; 8-OHdG: 8-hydroxy-2'deoxyguanosine; PP2A: Proteinphosphatase 2A; GSK-3ß: Glycogen synthase kinase-3ß; JNK: c-Jun Nterminal kinase; FBS: Fetal Bovine Serum; PBS: Phosphate-buffered saline; SDS: Sodium dodecyl sulfate; PVDF: polyvinylidene difluoride; TBS: Tris-buffered saline; DAPI: 4',6-diamidino-2-phenylindole.

\section{Declarations}

\section{Ethics approval and consent to participate}

Not applicable.

\section{Consent for publication}

Not applicable.

\section{Availability of data and materials}

The datasets used and/or analyzed during the current study are available from the corresponding author on reasonable request.

\section{Competing interests}

The authors declare no conflict of interest.

\section{Funding}


National Natural Science Foundation of China, 32060167, 81960265; Department of Education of Guizhou Province, KY[2021]204; Guizhou Science and Technology Department, [2019]1031, ZK[2021]357, LH[2017]7156, [2018]1009, [2020]1Y354; China Postdoctoral Science Foundation, 2020M683659XB;

Scientific Research Project of Guizhou University of Traditional Chinese Medicine, [2019]48; Science and Technology Bureau, Guiyang Municipal Government, [2019]9-2-7.

\section{Authors' contributions}

QC and ZT contributed to the conception and study design. QC, CC and FC performed the experiments. $\mathrm{CC}, \mathrm{FC}, \mathrm{YT}$ and $\mathrm{YY}$ contributed to data collection and data analysis. QC, CC, RN and ZT wrote the manuscript. All authors approved the final manuscript before submission.

\section{Acknowledgements}

Not applicable.

\section{References}

1. Kambe T, Tsuji T, Hashimoto A, Itsumura N: The Physiological, Biochemical, and Molecular Roles of Zinc Transporters in Zinc Homeostasis and Metabolism. Physiological Reviews 2015, 95(3):749784.

2. Sensi SL, Granzotto A, Siotto M, Squitti R: Copper and Zinc Dysregulation in Alzheimer's Disease. Trends Pharmacol Sci 2018, 39(12):1049-1063.

3. Mocchegiani E, Bertoni-Fre Dd Ari C, Marcellini F, Malavolta M: Brain, aging and neurodegeneration: Role of zinc ion availability. Progress in Neurobiology 2005, 75(6):367-390.

4. Miller Y, Ma B, Nussinov R: Zinc ions promote Alzheimer Abeta aggregation via population shift of polymorphic states. Proceedings of the National Academy of Sciences of the United States of America 2010, 107(21):9490-9495.

5. Deshpande A, Kawai H, Metherate R, Glabe CG, Busciglio J: A role for synaptic zinc in activitydependent Abeta oligomer formation and accumulation at excitatory synapses. J Neurosci 2009, 29(13):4004-4015.

6. Sensi SL, Ton-That D, Sullivan PG, Jonas EA, Weiss JH: Modulation of mitochondrial function by endogenous Zn2 + pools. Proceedings of the National Academy of Sciences 2003, 100(10):61576162.

7. Liu HY, Gale JR, Reynolds IJ, Weiss JH, Aizenman E: The Multifaceted Roles of Zinc in Neuronal Mitochondrial Dysfunction. Biomedicines 2021, 9(5):489.

8. Ji SG, Medvedeva YV, Wang H-L, Yin HZ, Weiss JH: Mitochondrial Zn2 + Accumulation: A Potential Trigger of Hippocampal Ischemic Injury. The Neuroscientist 2018, 25(2):126-138.

9. Zhao Y, Yan F, Yin J, Pan R, Shi W, Qi Z, Fang Y, Huang Y, Li S, Luo Y et al: Synergistic Interaction Between Zinc and Reactive Oxygen Species Amplifies Ischemic Brain Injury in Rats. Stroke 2018, 
49(9):2200-2210.

10. Pivovarova NB, Stanika RI, Kazanina G, Villanueva I, Andrews SB: The interactive roles of zinc and calcium in mitochondrial dysfunction and neurodegeneration. Journal of Neurochemistry 2014, 128(4):592-602.

11. Rivers-Auty J, Tapia VS, White CS, Daniels MJD, Drinkall S, Kennedy PT, Spence HG, Yu S, Green JP, Hoyle $C$ et al: Zinc Status Alters Alzheimer's Disease Progression through NLRP3-Dependent Inflammation. The Journal of Neuroscience 2021, 41(13):3025.

12. Dong X, Fu J, Yin X, Cao S, Li X, Lin L, Ni J: Emodin: A Review of its Pharmacology, Toxicity and Pharmacokinetics. Phytother Res 2016, 30(8):1207-1218.

13. Chao HW-H, Chen Y-K, Liu J-H, Pan H-T, Lin H-M, Chao H-M: Emodin protected against retinal ischemia insulted neurons through the downregulation of protein overexpression of $\beta$-catenin and vascular endothelium factor. BMC Complementary Medicine and Therapies 2020, 20(1):338.

14. Ahn SM, Kim HN, Kim YR, Choi YW, Kim CM, Shin HK, Choi BT: Emodin from Polygonum multiflorum ameliorates oxidative toxicity in HT22 cells and deficits in photothrombotic ischemia. Journal of Ethnopharmacology 2016, 188:13-20.

15. Li M, Fu Q, Li Y, Li S, Xue J, Ma S: Emodin opposes chronic unpredictable mild stress induced depressive-like behavior in mice by upregulating the levels of hippocampal glucocorticoid receptor and brain-derived neurotrophic factor. Fitoterapia 2014, 98:1-10.

16. Liu W, Fan Z, Gao F, Ou L, Li M, Zhou X, Luo W, Wei P, Miao F: Emodin inhibits zinc-induced neurotoxicity in neuroblastoma SH-SY5Y cells. Biosci Rep 2019, 39(5).

17. Li Z, Bi H, Jiang H, Song J, Meng Q, Zhang Y, Fei X: Neuroprotective effect of emodin against Alzheimer's disease via Nrf2 signaling in U251 cells and APP/PS1 mice. Molecular medicine reports 2021, 23(2).

18. Du C, Shi L, Wang M, Mao P, Wang J, Wei Y, Hou J, Wang M: Emodin attenuates Alzheimer's disease by activating the protein kinase $\mathrm{C}$ signaling pathway. Cellular and molecular biology (Noisy-le-Grand, France) 2019, 65(5):32-37.

19. Lai C, Chen Q, Ding Y, Liu H, Tang Z: Emodin protected against synaptic impairment and oxidative stress induced by fluoride in SH-SY5Y cells by modulating ERK1/2/Nrf2/HO-1 pathway. 2020, 35(9):922-929.

20. Schafe GE, Atkins CM, Swank MW, Bauer EP, Sweatt JD, LeDoux JE: Activation of ERK/MAP kinase in the amygdala is required for memory consolidation of pavlovian fear conditioning. $J$ Neurosci 2000 , 20(21):8177-8187.

21. Perry G, Roder H, Nunomura A, Takeda A, Friedlich AL, Zhu X, Raina AK, Holbrook N, Siedlak SL, Harris PL et al: Activation of neuronal extracellular receptor kinase (ERK) in Alzheimer disease links oxidative stress to abnormal phosphorylation. Neuroreport 1999, 10(11):2411-2415.

22. Pei JJ, Braak H, An WL, Winblad B, Cowburn RF, Iqbal K, Grundke-Iqbal I: Up-regulation of mitogenactivated protein kinases ERK1/2 and MEK1/2 is associated with the progression of neurofibrillary degeneration in Alzheimer's disease. Molecular Brain Research 2002, 109(1):45-55. 
23. Pei JJ, Gong CX, An WL, Winblad B, Cowburn RF, Grundke-lqbal I, Iqbal K: Okadaic-acid-induced inhibition of protein phosphatase $2 A$ produces activation of mitogen-activated protein kinases ERK1/2, MEK1/2, and p70 S6, similar to that in Alzheimer's disease. The American journal of pathology 2003, 163(3):845-858.

24. Lovell MA, Robertson JD, Teesdale WJ, Campbell JL, Markesbery WR: Copper, iron and zinc in Alzheimer's disease senile plaques. J Neurol Sci 1998, 158(1):47-52.

25. Tang Z, Bereczki E, Zhang H, Wang S, Li C, Ji X, Bran Ca RM, Lehtio J, Guan Z, Filipcik P: Mammalian Target of Rapamycin (mTor) Mediates Tau Protein Dyshomeostasis. Journal of Biological Chemistry 2013, 288(22):15556-15570.

26. Sun XY, Wei YP, Xiong Y, Wang XC, Xie AJ, Wang XL, Yang Y, Wang Q, Lu YM, Liu R et al: Synaptic released zinc promotes tau hyperphosphorylation by inhibition of protein phosphatase 2A (PP2A). The Journal of biological chemistry 2012, 287(14):11174-11182.

27. An WL, Bjorkdahl C, Liu R, Cowburn RF, Winblad B, Pei JJ: Mechanism of zinc-induced phosphorylation of p70 S6 kinase and glycogen synthase kinase 3beta in SH-SY5Y neuroblastoma cells. Journal of Neurochemistry 2005, 92(5):1104-1115.

28. Kim I, Park EJ, Seo J, Ko SJ, Kim CH: Zinc stimulates tau S214 phosphorylation by the activation of Raf/mitogen-activated protein kinase-kinase/extracellular signal-regulated kinase pathway. Neuroreport 2011, 22(16):839-844.

29. Huang Y, Wu Z, Cao Y, Lang M, Lu B, Zhou B: Zinc binding directly regulates tau toxicity independent of tau hyperphosphorylation. Cell reports 2014, 8(3):831-842.

30. Frazzini V, Granzotto A: The pharmacological perturbation of brain zinc impairs BDNF-related signaling and the cognitive performances of young mice. 2018, 8(1):9768.

31. Wang L, Yin YL, Liu XZ, Shen P, Wang JZ: Current understanding of metal ions in the pathogenesis of Alzheimer's disease. Translational Neurodegeneration 2020, 9(1).

32. Furuta T, Ohshima C, Matsumura M, Takebayashi N, Hirota E, Mawaribuchi T, Nishida K, Nagasawa $\mathrm{K}$ : Oxidative stress upregulates zinc uptake activity via Zrt/Irt-like protein 1 (ZIP1) in cultured mouse astrocytes. Life sciences 2016, 151:305-312.

33. Bossy-Wetzel E, Talantova MV, Lee WD, Schlzke MN, Lipton SA: Crosstalk between nitric oxide and zinc pathways to neuronal cell death involving mitochondrial dysfunction and p38-activated $\mathrm{K}+$ channels. Neuron 2004, 41(3):351-365.

34. Aizenman E, Stout AK, Hartnett KA, Dineley KE, Mclaughlin BA, Reynolds IJ: Induction of neuronal apoptosis by thiol oxidation: putative role of intracellular zinc release. Journal of Neurochemistry 2010, 75(5):1878-1888.

35. Liu W, Fan Z, Gao F, Ou L, Li M, Zhou X, Luo W, Wei P: Emodin inhibits zinc-induced neurotoxicity in neuroblastoma SH-SY5Y cells. 2019, 39(5).

\section{Figures}


(a)

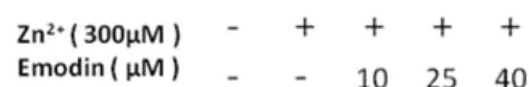

(b) (c)

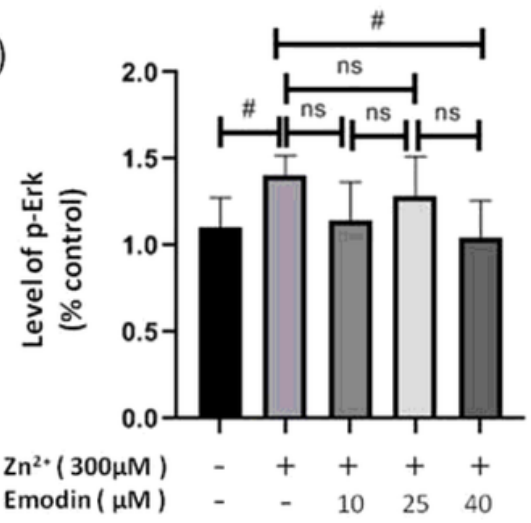

(d)

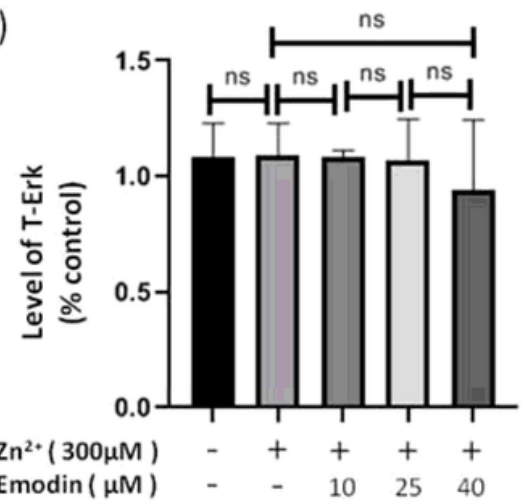

\section{Figure 1}

Emodin reversed the activation of the phosphorylated ERK1/2 in SH-SY5Y cells exposed to $300 \mu \mathrm{M}$ zinc sulfate. (a) Immunoblot image of phosphorylated ERK1/2 (p-Erk) and total ERK1/2 (t-ERK) in SH-SY5Y cells; (b) Stain-Free image of SH-SY5Y cell lysate; (c-d) Immunoblot analysis of phosphorylated ERK1/2 and total ERK1/2. Different concentrations of emodin (10, 25, and $40 \mu \mathrm{M})$ was applied. One-way ANOVA and Bonferroni post-hoc analysis, \#p $<0.05$; ns, not significant. Data as mean \pm SEM. 


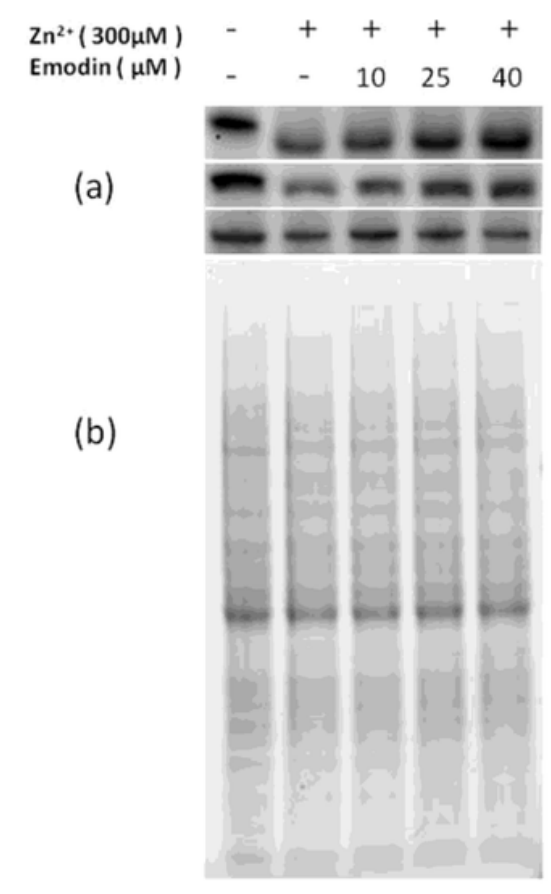

Synaptophysin

SNAP25

PSD95

Total protein (c)

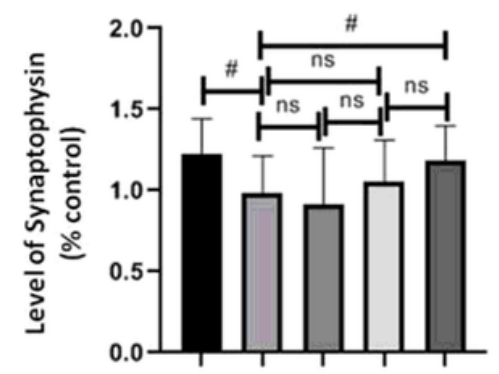

$2 n^{2+}(300 \mu M)$ $\operatorname{Emodin}(\mu \mathrm{M}) \quad-\quad-10 \quad 25 \quad 40$
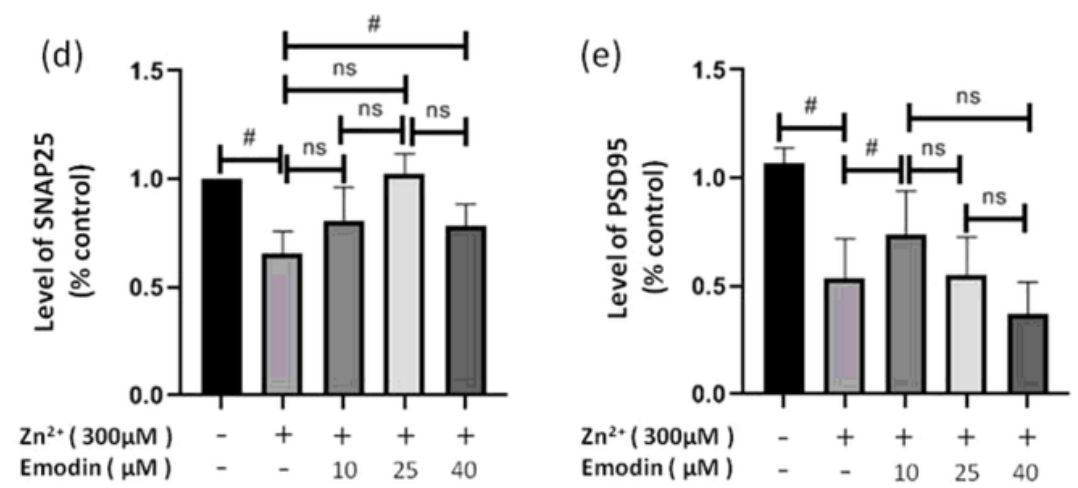

Figure 2

The dose-dependent effect of emodin on synapse-related proteins in SH-SY5Y cells exposed to $300 \mu \mathrm{M}$ zinc sulfate. (a) Immunoblots of Synaptophysin, SNAP25, and PSD95 in SH-SY5Y cells; (B) Stain-Free image of SH-SY5Y cell lysate; (c-e) Quantitation of the immunoblots. Different concentrations of emodin $(10,25$, and $40 \mu \mathrm{M})$ was applied. One-way ANOVA and Bonferroni post-hoc analysis, \#p < 0.05; ns, not significant; Data as mean \pm SEM. 
(a)
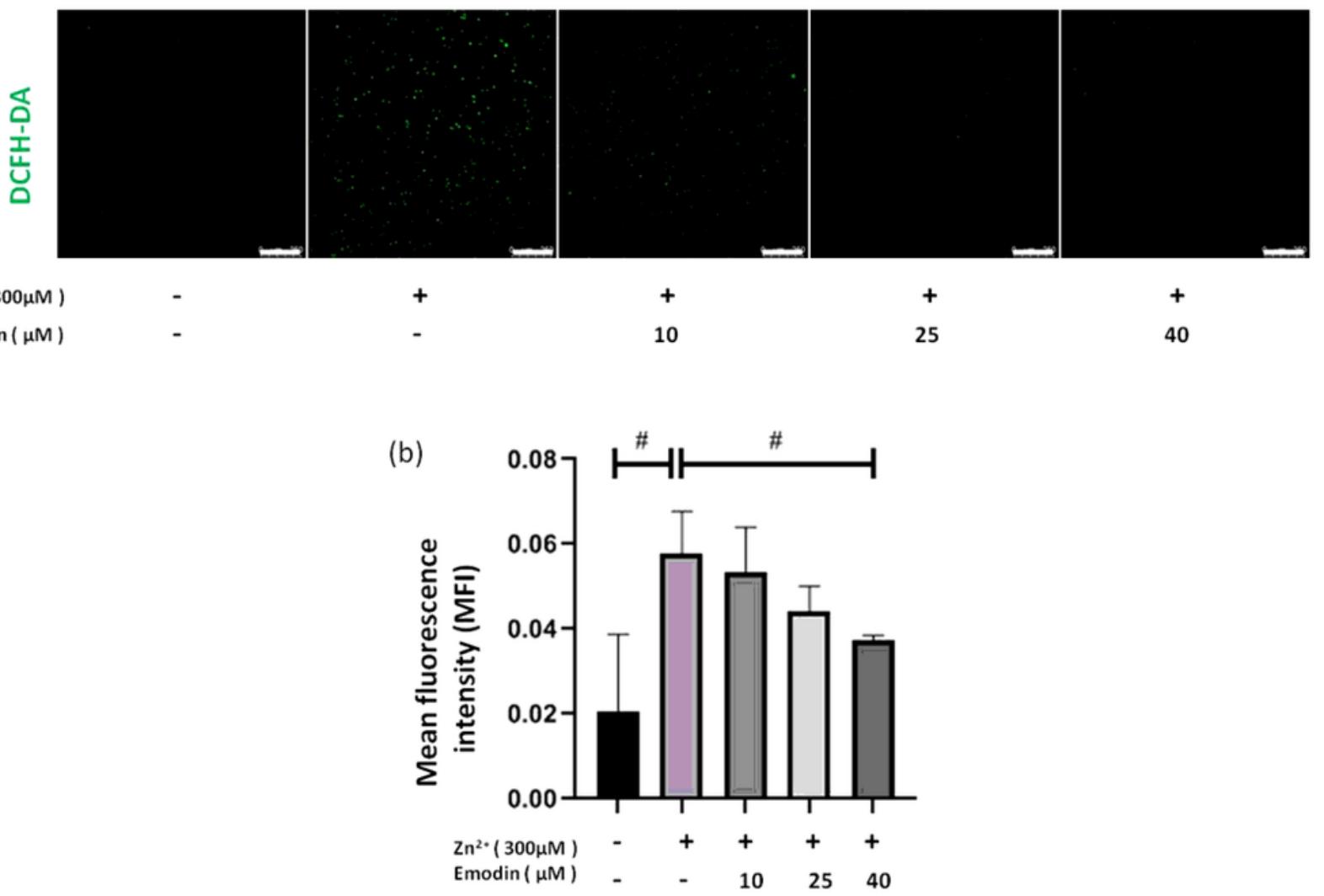

Figure 3

Emodin decreased the zinc-induced reactive oxygen species in SH-SY5Y cells. (a) fluorescence staining of DCFH-DA fluorescent staining in SH-SY5Y cells, from left to right are untreated cells, $300 \mu \mathrm{M}$ zinc sulfatetreated cells without emodin pretreatment $(10,25$, and $40 \mu \mathrm{M})$. scalebar $=250 \mu \mathrm{m}$. (b) the mean fluorescent intensity analysis. One-way ANOVA and Bonferroni post-hoc analysis, \#p < 0.05; ns, not significant. Data as mean \pm SEM. 

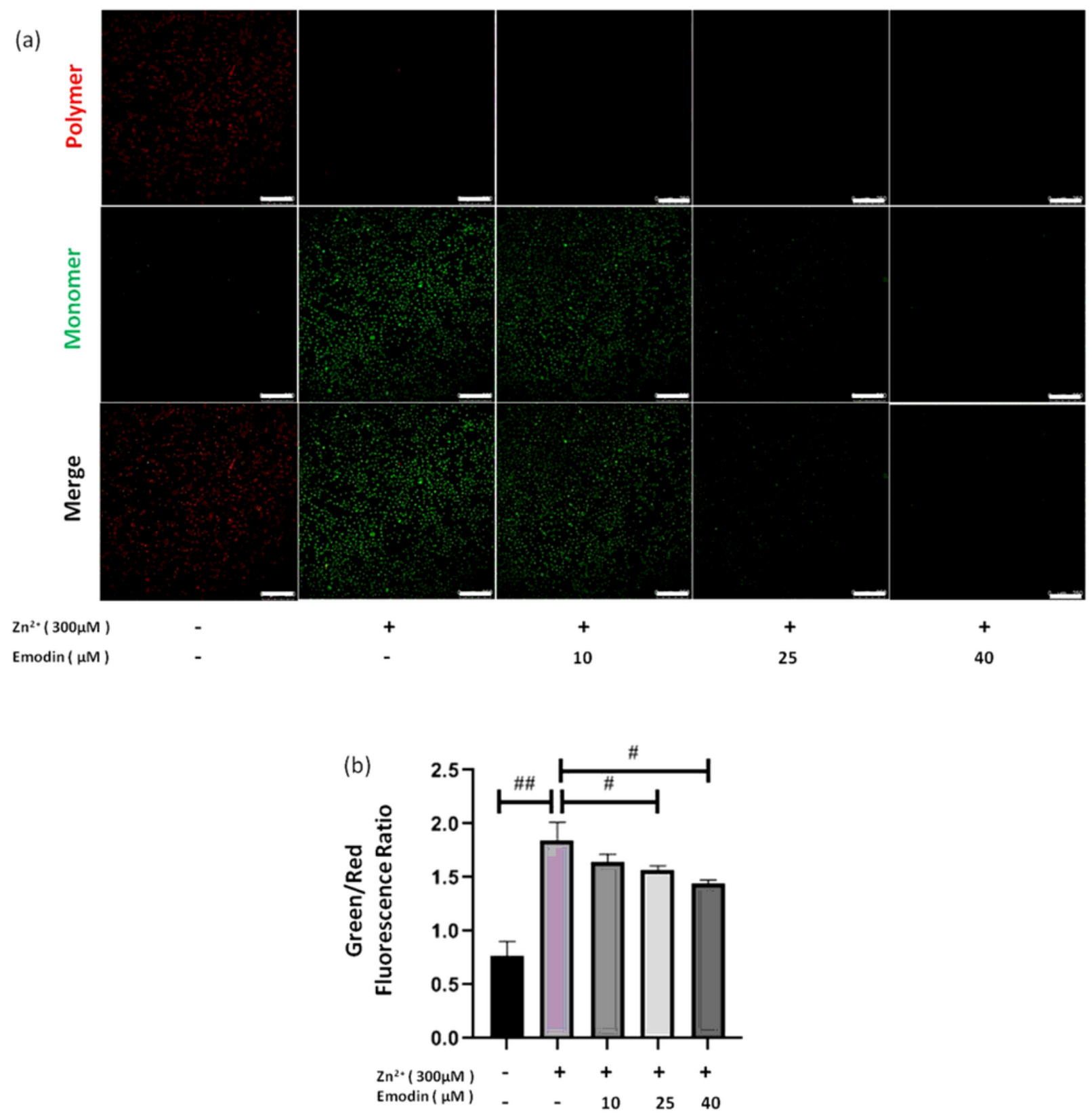

Figure 4

Emodin corrected the effects of zinc on depolarization of $\Delta \Psi \mathrm{m}$ in SH-SY5Y cells. (a) fluorescence staining of JC-1 in untreated and zinc-treated SH-SY5Y cells with or without pretreatment with emodin $(10,25$, and $40 \mu \mathrm{M})$. Mitochondrial aggregate, polymer form of JC-1 (red) indicating naormal $\Delta \Psi \mathrm{m}$, and monomeric form of $\mathrm{JC}-1$ (green) indicating dissipation of $\Delta \Psi \mathrm{m}$. scale bar $=250 \mu \mathrm{m}$. (b) the ratio of green fluorescence to red fluorescence. One-way ANOVA and Bonferroni post-hoc analysis; \#p < 0.05, \#\#p < 0.001 significant. Data as mean \pm SEM 

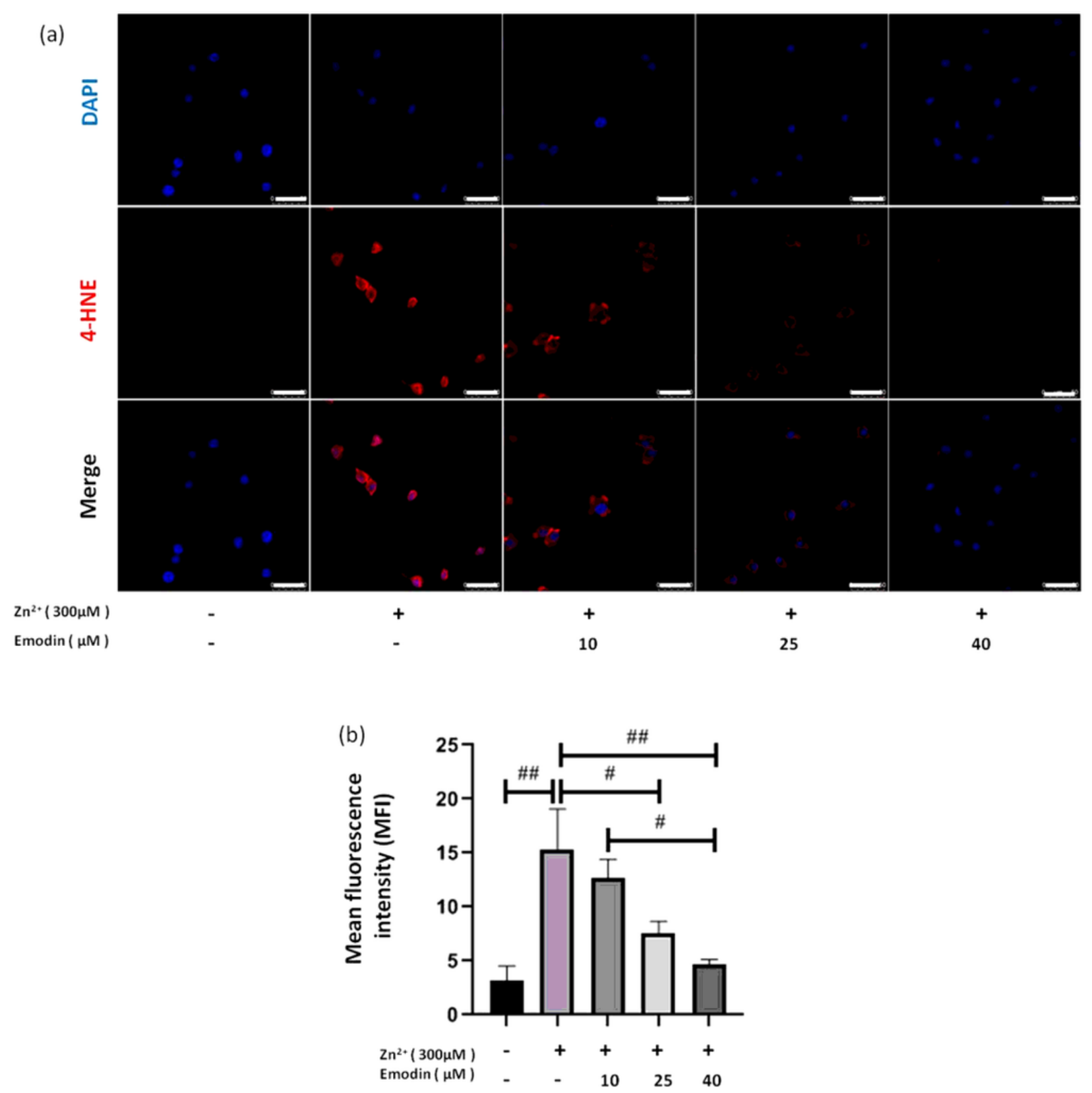

Figure 5

Emodin repressed lipid peroxidation in zinc-treated SH-SY5Y cells. (a) immunofluorescence-based confocal images of 4-Hydroxynonenal (4-HNE, red) in untreated and $300 \mu \mathrm{M}$ zinc sulfate -treated cells, with or without pretreatment with different concentrations of emodin with or without pretreatment with emodin $(10,25$, and $40 \mu \mathrm{M})$. scalebar $=50 \mu \mathrm{m}$; Nuclei was stained by DAPI (blue); (b) the mean fluorescent intensity analysis. One-way ANOVA and Bonferroni post-hoc analysis, $\# p<0.05, \# \# p<0.001$; Data as mean \pm SEM 


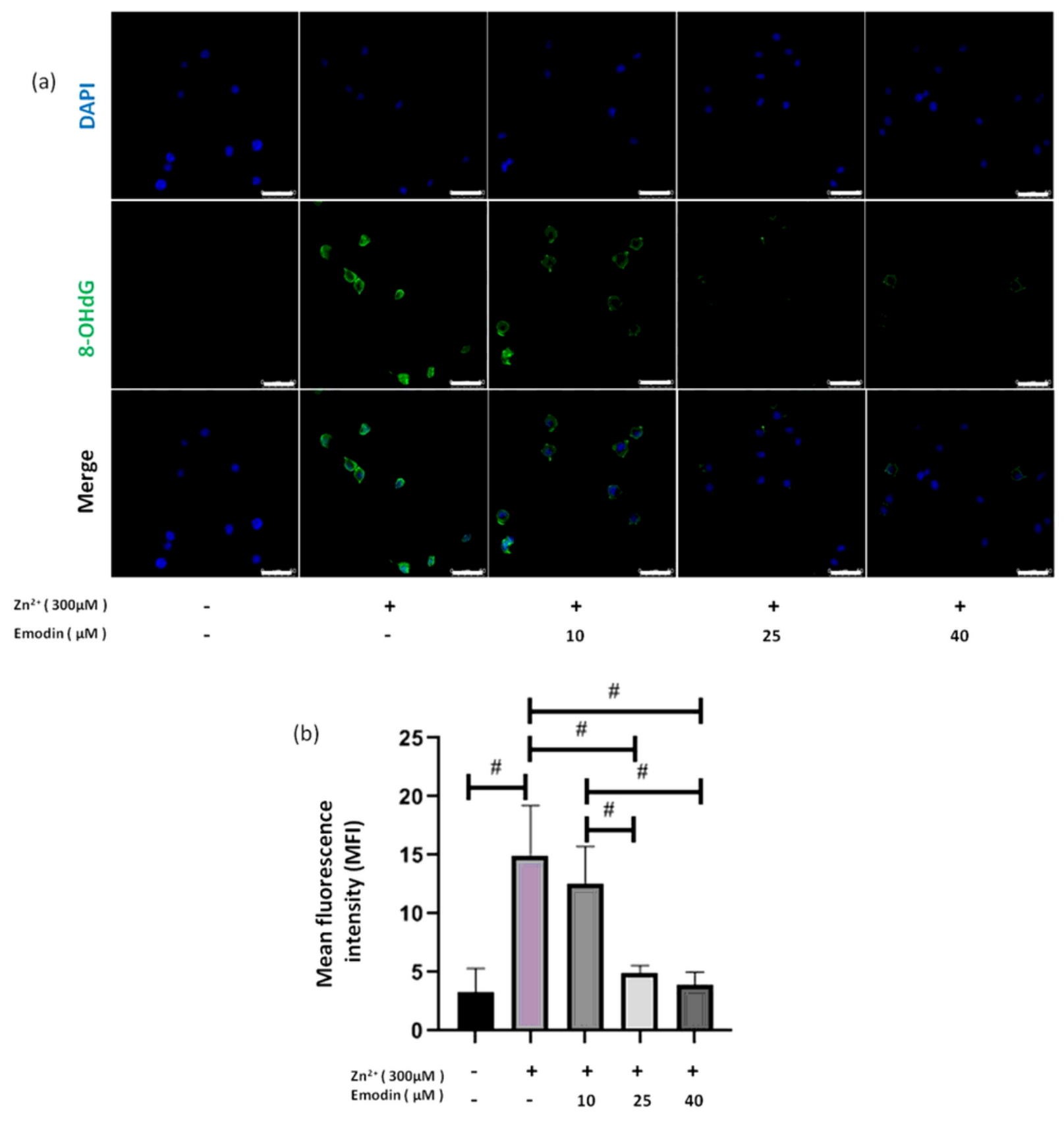

Figure 6

Emodin suppressed lipid peroxidation DNA oxidation in zinc-treated SH-SY5Y cells. (a) immunofluorescence-based confocal images showing the 8-hydroxy-2' -deoxyguanosine (8-OHdG, green) in untreated and $300 \mu \mathrm{M}$ zinc sulfate -treated cells with or without pretreatment with emodin $(10,25$, and $40 \mu \mathrm{M}$ ). scalebar $=50 \mu \mathrm{m}$; Nuclei was stained by DAPI (blue); (b) the mean fluorescent intensity analysis. One-way ANOVA and Bonferroni post-hoc analysis, \#p < 0.05; Data as mean \pm SEM.

\section{Supplementary Files}


This is a list of supplementary files associated with this preprint. Click to download.

- originaldata.pdf 